\title{
Commentary: Integrating urgent cardiovascular care delivery-An opportunity for meaningful change and collaboration
}

\author{
Gabriel S. Aldea, MD, FACS, FACC
}

\author{
From the Department of Cardiothoracic Surgery, University of Washington Medical School, Seattle, Wash. \\ Disclosures: Author has nothing to disclose with regard to commercial support. \\ Received for publication Jan 11, 2019; accepted for publication Jan 13, 2019; available ahead of print Feb 22, \\ 2019. \\ Address for reprints: Gabriel S. Aldea, MD, FACS, FACC, University of Washington Medical School, Cardiotho- \\ racic Surgery, Box 356310, 1959 NE Pacific St, Seattle, WA 98195-6310 (E-mail: aldea@uw.edu). \\ J Thorac Cardiovasc Surg 2020;159:551-12 \\ $0022-5223 / \$ 36.00$ \\ Copyright (c) 2019 by The American Association for Thoracic Surgery \\ https://doi.org/10.1016/j.jtcvs.2019.01.041
}

Transfer and care of patients in urgent condition remains a common major issue and highlights the fragmented and complex nature of current cardiovascular care delivery. It attempts to balance regional population-based and local needs with access, patient and family satisfaction, quality and safety, equity, financial and organizational sustainability. In the past 3 decades, acute coronary care systems and hospitals proliferated, with the aim of enhancing local access for early diagnosis of cardiovascular disorders and time-limited stabilization and therapy of acute coronary artery syndromes with percutaneous coronary intervention. These centers have been demonstrated to function safely without on-site cardiac surgery (NCS hospitals). These secondary NCS hospitals are a main portal for early diagnosis, triage, and referral to next-tier tertiary hospitals with cardiac surgical capabilities (CS hospitals) and to quaternary hospitals that provide broader regional care for more complex cardiovascular patients including those referred from tertiary CS hospitals (QCS hospitals). Although these systems have evolved and been in place for decades, there is a striking paucity of data to assess the safety and effectiveness of urgent transfers for cardiovascular surgical care. In this issue of the Journal, Beller and colleagues ${ }^{1}$ address an important aspect of this complex puzzle. They assess the effect of transfer status on outcomes of nonelective cardiac surgery (defined as hospital admission before surgical intervention). In the largest such study to date, which uses the Virginia Statewide Cardiac Services Quality Initiative (VCSQI), a Society of Thoracic Surgeons-based database that includes all 19 hospitals and surgical groups in the state, Beller and colleagues ${ }^{1}$ assessed demographics, risk factors, and outcomes of 13,094 patients treated from 2011 to 2017 undergoing Society of Thoracic Surgeons risk-stratified procedures-coronary artery bypass grafting $(84 \%)$, isolated valve operation $(8 \%)$, or valve operations plus coronary artery bypass grafting $(8 \%)$ - procedures that require admission before their surgical procedures.

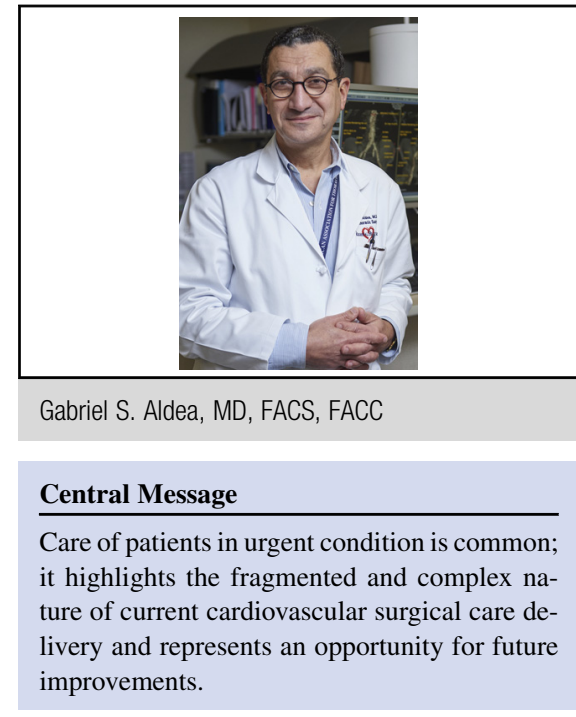

See Article page 540.

Transfers from NCS hospitals $(57.9 \%)$ and transfers from CS hospitals $(6.6 \%)$ are compared with patients admitted from the emergency department (33.5\%). Through multivariate analyses, Beller and colleagues ${ }^{1}$ concluded that patients admitted from the emergency department or transferred from NCS hospitals undergoing urgent cardiac surgery had similar preprocedural lengths of stay before their surgical intervention (no delays), with no adverse impact on either risk-adjusted mortality or morbidity. There was a statistically significant increase in hospital cost of about $\$ 6142$ related to stay other than in the intensive care unit (ICU), to nuanced differences in risk profiles and to added complexity of coordinating discharge planning for transferred patients. Patients transferred from CS hospitals to QCS hospitals had significantly higher predicted risk of mortality and morbidity, were more likely to present with congestive heart failure and to need redo surgery (4-fold) or valve surgery. Despite higher predicted risks of mortality and morbidity (1.6-fold) these patients had appropriate risk-adjusted observed to expected mortality ratio $(0.8$; $P=.55)$ and observed to expected morbidity ratio $(0.9$; $P=.35$ ), suggesting appropriate triaging and referral and excellent care for both transfer groups.

This publication is encouraging, because it definitively refutes previous concerns that were based on older, smaller 
studies that noted worse outcomes for patients transferred from other hospitals. ${ }^{2}$ Because of the acknowledged limitation of the database and limited scope of this publication, however, its conclusions are muted. It is evident that QCS hospitals have a better ability to provide 24-7 services, to stabilize and optimize the conditions of patients undergoing nonelective surgery, and to provide more sophisticated and nuanced diagnostic capabilities; have a broader spectrum of surgical expertise; have more extensive coordination of cardiac surgical and catheter-based therapy teams; and, finally, have more expert multidisciplinary perioperative care (both ICU and non-ICU care) to deliver these outcomes for patients with higher acuity and complexity compared to CS and NCS hospitals. Sustaining such units and expertise (specialization) is inherently expensive and requires complex staffing, coordination, high volumes, and financial resources. The calculated increased costs of caring for transferred patients noted in this publication are grossly underestimated. The costs of the immediate hospitalization before interhospital transfer and of interhospital medical transportation are significant and unaccounted for. In addition, there is also a significant unfunded human resource burden. Maintaining and staffing transfer centers, coordinating multiple patient hand-offs, and rebalancing variables volumes and patient throughput with fixed ICU, telemedicine units, and operating capacity are challenging and time-consuming, and they represent a significant underfunded burden and cost. The human strain factor is also underestimated. It is my conservative estimate that in my QCS hospital, 30\% of planned elective surgical procedures and schedules have to be altered in some manner to accommodate urgent and emergency transfers every week.

The incidence of urgent cardiac surgical care noted in this publication is strikingly high and underscores how common and important this issue truly is. One unstated opportunity is better triage of patients in the outpatient setting and earlier referral to appropriate NCS and QCS centers for elective evaluation and therapy according to objective anatomic and physiologic criteria, with a goal of decreasing urgent interventions with their higher associated acuity and morbidity. Another opportunity is addressing less common but much more complex elective and potentially morbid urgent conditions that are better cared for by QCS centers. If triaging can improve outcomes for urgent cardiac surgical procedures with lower predicted risk (such as isolated coronary artery bypass grafting or valve), we can only assume a potentially greater impact on patient survival for conditions that require a much higher level of specialization and coordinated care (such as acute aortic syndromes or cardiogenic shock). ${ }^{3,4}$ Demonstrating the value and impact of potential geospatial reorganization and initiatives does not always require multicenter trials; rather, these can be assessed by thoughtful modeling and simulation. ${ }^{5}$
Challenges and complexities of current cardiovascular care must not be underestimated. Longer-term regional planning has halted in this volatile time, highlighted by political gridlock and uncertainly that challenge fundamental aspects of health care delivery and financing. In addition, the US population is growing and aging, with the number of Americans older than 65 years expected to double in the next 3 decades, from 40 to 80 million. ${ }^{6,7}$ Despite preventive primary care therapies and strategies, the inexorable evolution in cardiovascular therapies will dramatically increase the acuity, complexity, and number of patients who will require cardiovascular care and has the potential to overwhelm current (limited) care delivery resources. ${ }^{8-11}$ To meet these unprecedented looming needs with a several-fold increase in volumes (and their associated increased cost), local NCS hospitals, CS hospitals, and QCS hospitals will need to evolve, be optimized, and, most importantly, be more formally and thoughtfully coordinated to provide high-quality, efficient, sustainable, and affordable care. Currently, regional cardiovascular care delivery is planned in an uncoordinated manner. Care is delivered by fragmented, competing, and frequently overlapping regional health care systems. Not surprisingly, planning focuses on short-term local needs and economic incentives, rather than optimal patient outcomes, let alone regional cost-effectiveness and sustainability.

As we recognize that a critical defining point and threshold has been reached, it is becoming more evident that smaller, incremental, iterative changes to our cardiovascular surgical care delivery may no longer suffice. This moment provides an opportunity for clear vision and for cardiovascular specialists to consider seriously and lead a more dramatic redesign in care delivery, one that is based on transparency, objective outcomes, efficiency, and utilization criteria. More responsive and robust communication, support, and coordination systems need to be created to help local physicians accurately assess and triage cardiovascular patients, with thoughtful sharing and integration of initial as well as longer-term care. Such efforts should aim to view and optimize the use of all current health care delivery personnel and different hospitals systems in a way that recognizes the unique roles, contributions, and responsibilities that each component has in this shared, informal, and interdependent network.

\section{References}

1. Beller JP, Hawkins RB, Mehaffey JH, Chancellor WZ, Fonner CE, Speir AM, et al. Impact of transfer status on real-world outcomes in nonelective cardiac surgery. J Thorac Cardiovasc Surg. 2020;159:540-50.

2. Prabhu S, Blusztein D, Jackson D, Sharma M, Arunothayaraj S, Stokes MB, et al. The effect of treatment delays associated with inpatient inter-hospital transfer from peripheral to tertiary hospitals for the surgical treatment of cardiology patients. Heart Lung Circ. 2016;25:75-81.

3. Harris DG, Olson SB, Rosen CB, Kalsi R, Taylor BS, Diaz JJ, et al. Early treatment at a referral center improves outcomes for patients with acute vascular disease. Ann Vasc Surg. 2018;50:52-9. 
4. Chakravarthy M, Tsukashita M, Murali S. A targeted management approach to cardiogenic shock. Crit Care Clin. 2018;34:423-37.

5. Concannon TW, Kent DM, Normand SL, Newhouse JP, Griffith JL, Ruthazer R, et al. A geospatial analysis of emergency transport and interhospital transfer in ST-segment elevation myocardial infarction. Am J Cardiol. 2008;101:69-74.

6. Centers for Disease Control and Prevention (CDC). Trends in aging-United States and worldwide. MMWR Morb Mortal Wkly Rep. 2003;52:101-4. 106

7. Ortman JM, Velkoff VA, Hogan H. An aging nation: the older population in the United States. Available at: https://www.census.gov/prod/2014pubs/p25-1140. pdf. Accessed February 13, 2019.
8. Heron M, Hoyert DL, Murphy SL, Xu J, Kochanek KD, Tejada-Vera B. Deaths: final data for 2006. Natl Vital Stat Rep. 2009;57:1-134.

9. Moore M, Chen J, Mallow PJ, Rizzo JA. The direct health-care burden of valvular heart disease: evidence from US national survey data. Clinicoecon Outcomes Res. 2016;8:613-27.

10. Martini EM, Garrett N, Lindquist T, Isham GJ. The boomers are coming: a total cost of care model of the impact of population aging on health care costs in the United States by major practice category. Health Serv Res. 2007;42(1 Pt 1): 201-18.

11. Rice DP, Fineman N. Economic implications of increased longevity in the United States. Annu Rev Public Health. 2004;25:457-73. 